\title{
Comparación entre Etil-cianoacrilato y Sutura Convencional en el cierre de incisiones de Piel de Conejo (Oryctolagus cuniculus)
}

\author{
Comparison between Ethyl-cyanoacrylate and Conventional Suture for the \\ Closure of Skin Incisions in Rabbit (Oryctolagus cuniculus)
}

"Vásquez, B.; ${ }^{* *}$ Schencke, C.; ${ }^{* * *}$ Rodríguez, C.; ${ }^{* * *}$ Veuthey, C. \& ${ }^{* * * *}$ del Sol, M.

VÁSQUEZ, B.; SCHENCKE, C.; RODRÍGUEZ, C.; VEUTHEY, C. \& DEL SOL, M. Comparación entre Etil-cianoacrilato y sutura convencional en el cierre de incisiones de piel de conejo (Oryctolagus cuniculus). Int. J. Morphol., 30(3):797-802, 2012.

RESUMEN: Las ventajas de los adhesivos en base a cianoacrilatos para uso en cierre de heridas cutáneas ha sido demostrada, pero escasos estudios reportan el uso del etil-cianoacrilato a nivel nacional. El objetivo fue comparar aspectos morfológicos de cicatrización en piel de conejo entre etil-cianoacrilato y sutura. Utilizamos 10 conejos machos adultos, divididos en 2 grupos de 5 animales, del Centro de Cirugía Experimental de la Universidad de La Frontera, Chile. Al grupo experimental se le realizaron dos incisiones, una en cada lado del dorso del tórax, $4 \mathrm{~cm}$ de largo, atravesando epidermis y dermis. La incisión derecha fue cerrada con etil-cianoacrilato, la izquierda con punto intradérmico de ácido poliglicólico. Se tomaron biopsias a los días 7, 14 y 21 días postoperatorio. Al día 7, la incisión tratada con etil-cianoacrilato presentó una reacción inflamatoria moderada, con infiltración moderada de PMN. Las biopsias de sutura presentaban una etapa de regeneración más avanzada, sin presentar desarrollo epidérmico. Al día 14, las incisiones tratadas con etilcianoacrilato se encontraban en la fase final de la etapa inflamatoria, mientras que las biopsias de sutura se encontraban ya en etapa proliferativa. Al día 21, ambos tipos de cierre se encontraban en etapa de remodelación, con epidermis gruesa y lámina basal formada. La dermis presentó tejido conectivo denso irregular, escasos folículos pilosos y glándulas sebáceas. El etil-cianoacrilato, como adherente cutáneo, es una buena alternativa de uso, mostrando una adecuada biocompatibilidad. Presentando una reacción inflamatoria moderada, limitada y de corta duración a nivel dérmico. La cicatriz logra mejores resultados estéticos con respecto a las incisiones cerradas con sutura. Se debe considerar su uso limitado a sitios no expuestos a movimientos y de baja tensión.

PALABRAS CLAVE: Conejo; cicatrización; etil-cianoacrilato; sutura.

\section{INTRODUCCIÓN}

Una lesión en la piel que altere la continuidad de la superficie cutánea desencadena los mecanismos de reparación. Según Cardoso (2003), Benavides (2008), la Sociedad Argentina de Dermatología (2008) y Ramírez (2010), los procesos fisiológicos de reparación de heridas pueden ser divididas en tres fases: inflamatoria (dividida en hemostática y celular), proliferativa y de remodelación, estas fases no son mutuamente excluyentes, sino, sobrepuestas en el tiempo. La fase inflamatoria hemostática se inicia al momento de la lesión y se caracteriza por la activación plaquetaria; la fase celular se inicia con la aparición de neutrófilos, permaneciendo en heridas no infectadas, siendo la fuente principal de citoquinas proinflamatorias o interleuquinas y TNF. Estas células son reemplazadas por macrófagos, que son considerados claves en la transición entre la fase inflamatoria y proliferativa. La fase proliferativa o de granulación, presenta angiogénesis y fibroplasia dérmica, junto con el inicio de la epitelización de la herida. La fase de remodelación es la última etapa, se inicia por la fibroplasia y continúa por meses según el tipo de herida, remodelando la colágena, matríz extra celualar (MEC) y epidermis.

Con el fin de mejorar el proceso de cicatrización en heridas traumáticas o quirúrgicas, se han desarrollado diversos materiales de sutura y compuestos adhesivos. El cianoacrilato, un biomaterial sintético, tiene la propiedad de endurecerse en presencia de los fluidos biológicos y adherirse fuertemente a los tejidos. Es un producto biodegradable que presenta propiedades hemostáticas y bactericidas. Alquil-2-cianoacrilatos fue primero sintetiza-

\footnotetext{
Facultad de Ciencias de la Salud, Universidad Autónoma de Chile, Sede Temuco, Chile.

* Doctorado en Ciencias Morfológicas, Universidad de La Frontera, Temuco, Chile.

*** Universidad Mayor, Sede Temuco, Chile.

${ }^{* * * *}$ Facultad de Medicina, Universidad de La Frontera, Temuco, Chile.
} 
do en 1949, siendo usado luego para cerrar heridas en soldados durante la $2^{\circ}$ Guerra Mundial. El cianoacrilato es un adhesivo líquido, se aplica en forma tópica, polimerizando en aproximadamente 10 segundos. Se ha empleado en múltiples áreas de la medicina, cierre de fístula esofágica, tratamiento quirúrgico en miocardio, mamoplasia bilateral y cierre facial (Orozco-Razón et al., 2002 y Shivamurthy et al., 2010).

Los diferentes tipos de cianoacrilatos se logran alterando la cadena lateral, obteniendo metil $(\mathrm{R}=\mathrm{CH} 3)$, etil ( $\mathrm{R}=\mathrm{C} 2 \mathrm{H} 5)$, butil $(\mathrm{R}=\mathrm{C} 4 \mathrm{H} 9)$ y octil-cianoacrilato ( $\mathrm{R}=\mathrm{C} 8 \mathrm{H} 17)$. Su toxicidad está relacionada con su velocidad de degradación y tamaño de la cadena. Así, el metilcianoacrilato demostró ser tóxico en animales a dosis farmacológicas, causando edema y necrosis residual, siendo por lo tanto, contraindicado para su uso clínico (Faion, 2009). Los cianoacrilatos de cadena larga son en cambio, derivados que carecen de toxicidad, con una elaboración más sofisticada (Dalvi et al.,1986; Borba et al., 2000; FreitasJunior et al., 2008 y Reyes et al., 2010).

Montanaro et al. (2001), describen la citotoxicidad del etil-cianoacrilato, con lisis celular observada in vitro, con intensa reacción inflamatoria in vivo, atribuyendo la toxicidad a los productos de degeneración, formaldehidocianoacetato. Sin embargo, más recientemente Souza \& Briglia (2011), en estudios realizados en ratas, señalaron que el etil-cianoacrilato no es cancerígeno, no genera reacciones alérgicas y no genera efectos citotóxicos.

Este adhesivo se ha utilizado en clínica para Medicina Humana y Veterinaria (Andrade et al., 2001), siendo recomendado sólo para uso en superficies avasculares como la piel (Souza et al., 2007). Saska et al. (2009), demostró que el adhesivo Super Bonder® (etil-cianoacrilato) promueve una reacción inflamatoria de baja intensidad en el tejido subcutáneo de ratas, siendo menor que con el Histacril@ (butil-cianoacrilato), sin generar necrosis residual.

Por otra parte, la evolución de la sutura ha llegado a tal grado de refinamiento que incluye materiales especialmente diseñados para cada procedimiento específico (Chicas, 2004; Reyes et al., 2010).

A pesar de las sofisticaciones de los materiales actuales y de las técnicas quirúrgicas, cerrar una herida es todavía objeto de estudio en cuanto a sus beneficios de acción mecánica, biocompatibilidad y costo-eficiencia. El etilcianoacrilato es un adhesivo eficiente en el cierre de heridas, y de menor costo si lo comparamos con otros cianoacrilatos (Souza \& Briglia), sin embargo, aún son pocos los trabajos que promuevan su uso clínico. Por este motivo, es necesario analizar la acción del etil-cianoacrilato a nivel epidérmico y dérmico, simplicidad de uso, posibilidad de infección y resultado estético. El objetivo de nuestro estudio fue comparar aspectos histológicos entre etilcianoacrilato y sutura convencional en el cierre de incisiones de piel de conejo (Oryctolagus cuniculus).

\section{MATERIAL Y MÉTODO}

Utilizamos 10 conejos machos (Oryctolagus cuniculus) adultos, sanos, mantenidos con pellet ad libitum en el Centro de Cirugía Experimental de la Universidad de La Frontera, Temuco, Chile. Los animales fueron divididos en grupos de 5 conejos. Al grupo de estudio, se le realizó dos incisiones, uno en el dorso derecho del tórax y otro en el dorso izquierdo del tórax, post tricotomía y asepsia de la zona quirúrgica. Cada incisión fue de $4 \mathrm{~cm}$ de largo, atravesando epidermis y dermis, sin llegar a la musculatura. Posteriormente, la incisión del lado derecho fue cerrada con pegamento de etil-cianoacrilato (Agorex ${ }^{\circledR}$ Instantáneo líquido precisión) y la del lado izquierdo cerrada con punto intradérmico de ácido poliglicólico 2/0. Al grupo control, solo se le tomó un biopsia de piel. Durante el procedimiento quirúrgico, los animales fueron anestesiados vía intramuscular con Xilazina, Ketamina y Atropina en dosis de $5 \mathrm{mg} / \mathrm{Kg}, 35 \mathrm{mg} / \mathrm{kg}$ y $0,045 \mathrm{mg} / \mathrm{kg}$ respectivamente.

Del grupo experimental, se tomaron biopsias de las incisuras cerradas con pegamento y sutura los días 7, 14 y 21 postoperatorio. Las muestras fueron fijadas en formalina tamponada al $10 \%$, deshidratadas e incluidas en parafina para obtener cortes histológicos de $4 \mu \mathrm{m}$ de grosor. Para el análisis histológico las secciones fueron teñidas con HE, tricómico de Masson (TCM) e impregnación de plata para fibras reticulares. Para el análisis histoquímico, utilizamos ácido periódico de Schiff (PAS). Las láminas fueron observadas en un microscopio óptico Olympus ${ }^{\circledR}$ modelo CX31, con cámara marca Moticam ${ }^{\circledR}$ modelo 480. Las imágenes fueron proyectadas en un monitor de pantalla plana marca Sony®. Se evaluó el grosor de la epidermis en la zona cicatrizal el día 21 post cirugía, para ello se utilizó el programa AxioVision LE 4.8.2.0 Se determinó además, en un recuento total de 100 células en la zona cicatrizal, el porcentaje de polimorfonucleares (PMN) y mononucleares (MN) en los días 7 y 14 post cirugía.

\section{RESULTADOS}

Al día 7 post cirugía, las biopsias de incisiones cerradas con etil-cianoacrilato presentaron una etapa 
inflamatoria, sin desarrollo de epidermis. A nivel dérmico se observó un tejido conectivo edematoso en las zonas próximas a la lesión, con presencia de un 79,67\% de PMN y un 20,33\% de MN, neoformación de vasos sanguíneos y formación de fibras colágenas inmaduras. Los fibroblastos presentaron núcleo ovoide, ligeramente aplanado y levemente hipertrófico (Fig. 1A). Las biopsias de incisión cerrada con sutura de ácido poliglicólico $2 / 0$ presentaron una etapa inflamatoria más avanzada, sin reepitelización de la zona lesionada. La epidermis cercana a la incisión se observó con bordes gruesos e irregulares y la dermis presentó menor formación de edema. Se observó un 31,67\% de PMN y 68,33\% de MN, neoformación de vasos sanguíneos y tejido conectivo denso irregular en la zona cicatrizal (Fig.1B).
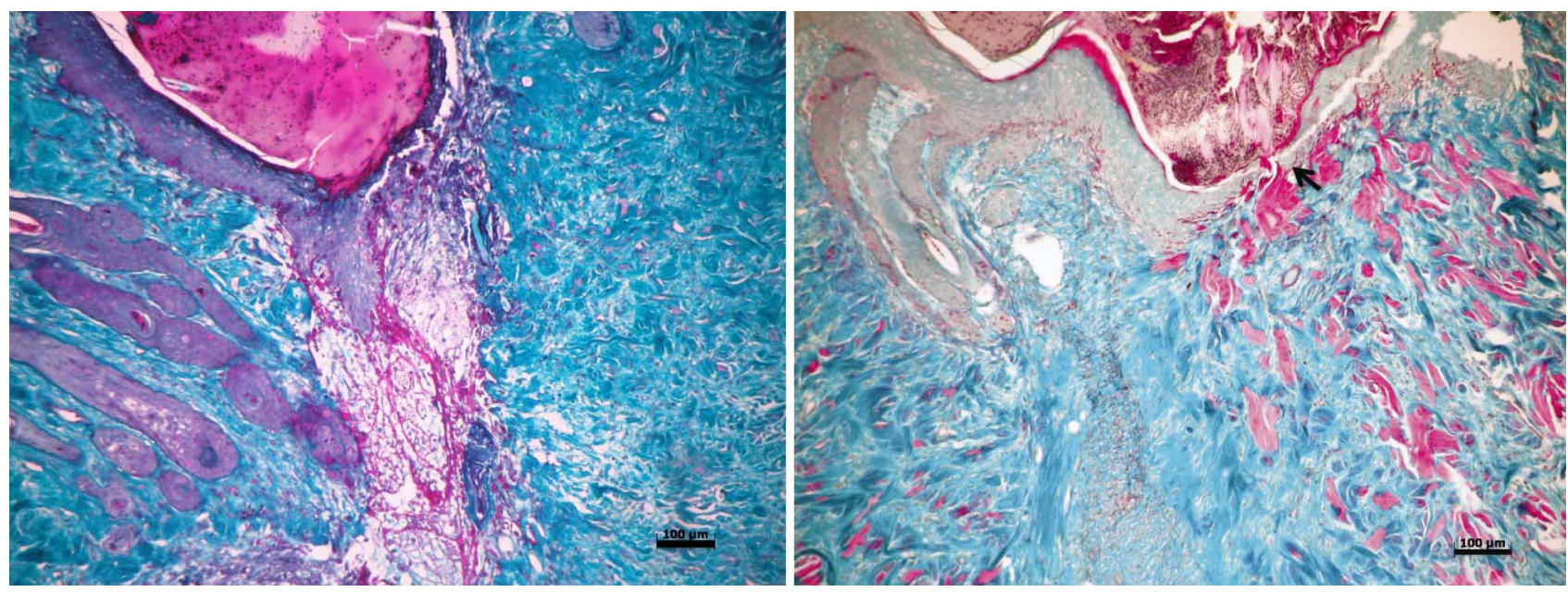

Fig. 1. Día 7 post cirugía, incisión cerrada con etil-cianoacrilato (A) y sutura (B), tinción Tricrómico de Masson. A. La dermis en regeneración presentó fibras colágenas inmaduras y escasa presencia de MEC, sin regeneración de epidermis. Se observa la presencia de escara hacia el lumen. B. Epidermis en estado inicial de desarrollo (flecha), la dermis presentó tejido conectivo denso. Hacia el lumen se observa la presencia de escara.
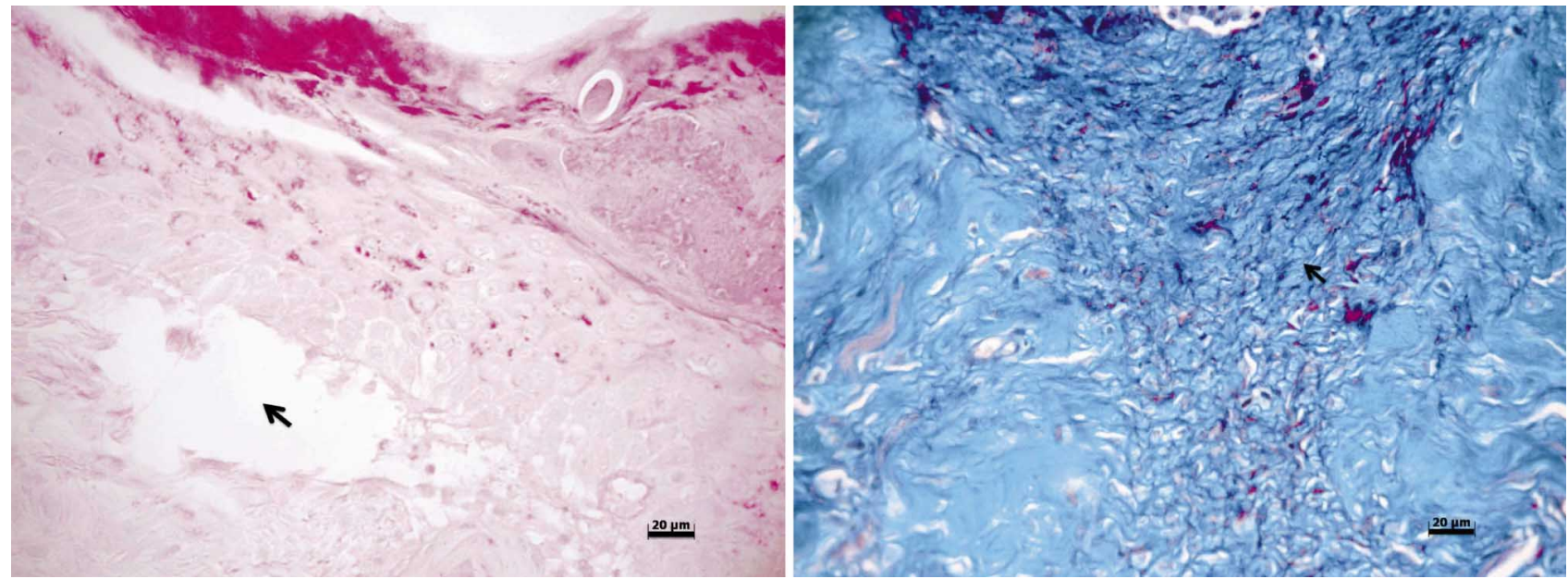

Fig. 2. Día 14 post cirugía, incisión cerrada con etil-cianoacrilato (A) y sutura (B). A. La epidermis aún no se regenera por completo y la lámina basal se presenta discontinua. A nivel dérmico se observa presencia de edema (flecha). B. La dermis presenta tejido conectivo denso irregular, fibras colágenas y reticulares que cubren toda la zona en regeneración (flecha). 

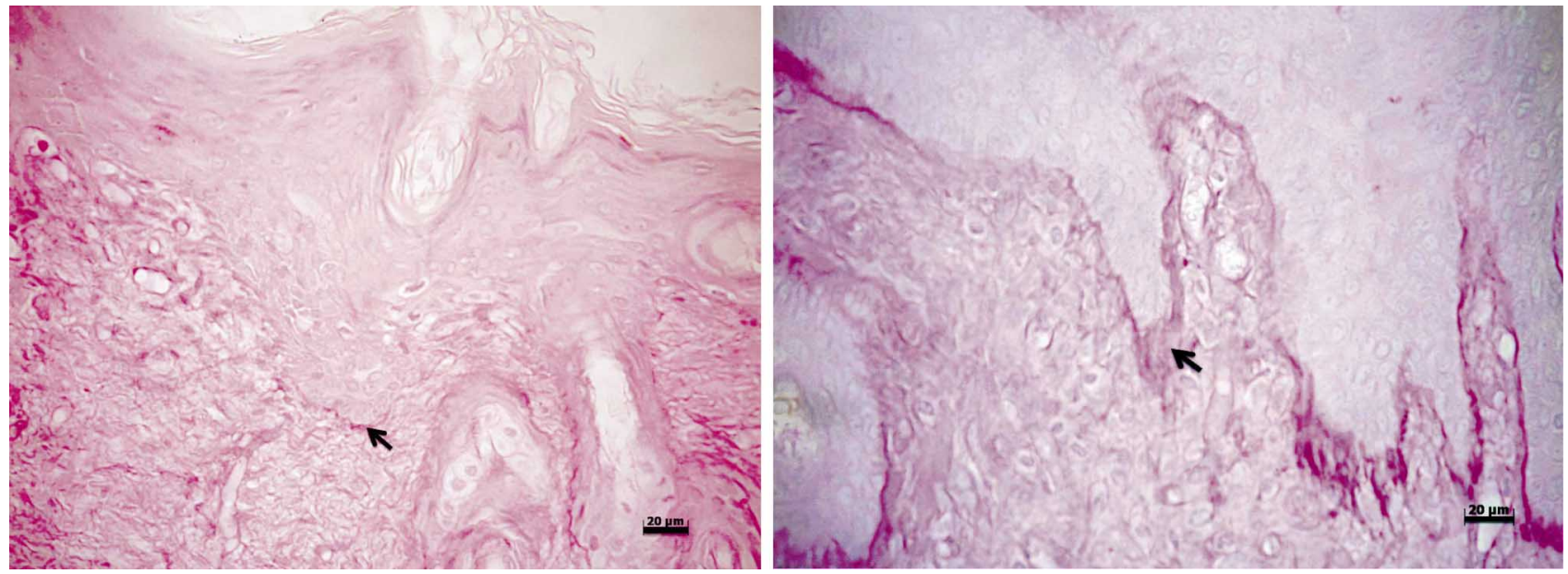

Fig. 3. Día 21 post cirugía, incisión cerrada con etil-cianoacrilato (A) y sutura (B), tinción de PAS. A. Se observa una gruesa epidermis queratinizada, con presencia de lámina basal (flecha). B. La biopsia evidencia una capa epidérmica gruesa e irregular, con lámina basal evidente (flecha).

Al día 21 post cirugía, las muestra cerradas con etilcianoacrilato presentaron una etapa de remodelación, cuya zona cicatrizal presentó una epidermis gruesa y altura promedio de 71,95 mm (DE 24,61). La lámina basal fue visible mediante la tinción PAS (Fig. 3A). La dermis presentó un tejido conectivo denso irregular, con escasa presencia de folículos pilosos y glándulas sebáceas. En las incisiones cerradas con sutura, la zona cicatrizal también presentó una etapa de remodelación, mostrando una epidermis gruesa e irregular en la zona cicatrizal y áreas contiguas, con una altura promedio de 104,68 mm (DE 55,86). Se observó una lámina basal de reacción intensa para la tinción de PAS. La dermis presentaba algunas glándulas sebáceas, con ausencia de folículos pilosos. El tejido conectivo se observó denso e irregular (Fig. 3B).

En ambas técnicas de cierre utilizadas, se observó el desarrollo de papilas dérmicas. La altura epidérmica de la biopsia control fue en promedio 38,25 mm (DE 9,24).

\section{DISCUSIÓN}

En nuestro estudio, el etil-cianoacrilato presentó resultados satisfactorios, sin necrosis del tejido lesionado, ni efectos adversos o complicaciones cosméticas en el cierre de las heridas, concordando con lo observado en investigaciones realizadas en ratas por Borba et al., Freitas-Junior $e t$ $a l$. y Souza \& Briglia, en perros por Sangwan et al., 2003 y Vasconcellos et al., 2005 y evaluaban en humanos por Montes et al., 2009.
La reacción inflamatoria por el uso de etilcianoacrilato fue moderada, de acuerdo a los análisis histológicos e histoquímicos observados. La infiltración de PMN en incisiones cerradas con etil-cianoacrilato presentó mayor porcentaje tanto para el día 7 (79,67\%) como para el día $14(63,33 \%)$ comparado con incisiones cerradas con sutura. Por el contrario, la presencia de MN fue mayor en incisiones cerradas con sutura para los días $7(68,33 \%)$ y 14 $(84,67 \%)$ post cirugía comparada con las incisiones cerradas con etil-cianoacrilato. Este hecho podría explicar porqué el proceso de reparación de la heridas cerradas con etilcianoacrilato fueron más lentas que las suturas para los días 7 y 14 post cirugía. Los MMN tales como los macrófagos se observan en etapas más avanzadas de la inflamación, participan regulando la llegada de monocitos y fibroblastos, liberando factores angiogénicos y de crecimiento endotelial vascular, los cuales son importantes para la migración y proloferación celular y formación de la MEC (Benavides y Ramírez). Sin embargo, al día 21 post cirugía ambos tipos de cierre de heridas se encontraban terminando la etapa proliferativa e iniciando la fase de remodelación. En esta etapa no se observó signos de inflamación, coincidiendo con lo señalado por Souza et al.

Toriumi et al., 1990 y Montanaro et al., señalaron que la desventaja de los cianoacrilatos es su citohistotoxicidad, indicando que el etil-cianoacrilato presentaba una reacción histotóxica moderada comparada con otros cianoacrilatos de cadena corta. Sin embargo, sus efectos adversos han sido aceptados ya que éstos son locales, especialmente por su inflamación y dehiscencia parcial. Andrade et al., Saska et al. y Souza \& Briglia no observaron necrosis 
a nivel dérmico y baja infiltración de neutrófilos. En nuestro estudio, al día 14 post cirugía, la presencia de tejido granular y fibrosis fue similar a la esperada en un proceso normal de cicatrización en etapa proliferativa.

La altura epidérmica en las incisuras cerradas con sutura, alcanzó 104,68 mm, mayor a lo observado con etilcianoacrilato, cuya altura fue $71,95 \mathrm{~mm}$, observándose menos pliegues epidérmicos y logrando una cicatriz con mejores resultados estéticos, similar a lo obtenido en otros estudios comparativos (Sangwan et al., Souza et al. y Montes et al.).

El tiempo requerido para tratar el cierre de las heridas con etil-cianoacrilato fue la mitad del tiempo utilizado con sutura, concordando con otros autores (Reyes et al., Freitas-Junior et al., Dalvi et al. y Carneiro et al.), quienes indicaron que este tipo de adhesivo promueve una hemostasis inmediata, simplificando y acortando el procedimiento operatorio, sin interferir en el proceso de reparación, observarse supuración, o necrosis residual de la herida.
Carneiro et al., observaron en incisiones de piel de ratas, cerradas con etil y butil-cianoacrilato, que cuanto mayor es la cadena lateral del cianoacrilato, menor es la histotoxicidad, pero también menor su adhesividad y mayor el tiempo de curación. Por su parte, Souza et al., señalaron que el etil-cianoacrilato fue ventajoso sobre el octylcianoacrilato y sutura, presentando menos complicaciones en el cierre de incisiones en piel de rata.

Finalmente, podemos concluir que el etilcianoacrilato presentó mayor eficacia sobre el método de sutura tradicional en el cierre de heridas, mostrando ventajas tales como, fácil aplicación tópica, polimerización rápida (menor a 10 segundos), resistencia eficaz para afrontar los bordes de la herida en piel, respuesta inflamatoria aceptable, cicatriz de buena calidad estética y bajo costo. Sin embargo, el uso de etil-cianoacrilato estará finalmente determinado por el conocimiento de sus ventajas y desventajas, e indicaciones específicas por parte del profesional de la salud o del área veterinaria.

VASQUEZ, B.; SCHENCKE, C., RODRIGUEZ, C.; VEUTHEY, C. \& DEL SOL, M. Comparison between Ethyl-cyanoacrylate and conventional suture for the closure of skin incisions in rabbit (Oryctolagus cuniculus). Int J. Morphol., 30(3):797-802, 2012.

SUMMARY: The advantages of cyanoacrylate based adhesives for use in cutaneous wound closure has been demonstrated, but few studies reporting the use of ethyl cyanoacrylate nationwide. The objective was to compare morphological aspects of healing in rabbit skin between ethyl-cyanoacrylate and suture. We used 10 adult male rabbits divided into 2 groups of 5 animals, the Center for Experimental Surgery, Universidad de La Frontera, Chile. The experimental group was subject to two incisions, one at each side of the dorsum of the thorax, $4 \mathrm{~cm}$ long, spanning epidermis and dermis. The incision was closed with ethyl right-cyanoacrylate, intradermal left with point of polyglycolic acid. Biopsies were taken at days 7, 14 and 21 days postoperatively. At day 7, the incision treated with ethyl cyanoacrylate presented a mild inflammatory reaction with moderate infiltration of PMNs. Suture biopsies showed a more advanced stage of regeneration, without presenting epidermal development. At day 14, the incisions treated with ethyl cyanoacrylate were in the final phase of the inflammatory stage, while suture biopsies were already in proliferative stage. At day 21 , closing both types were remodeling stage, with thicker epidermis and basal lamina formed. The dermis showed irregular dense connective tissue, poor hair follicles and sebaceous glands. Ethyl-cyanoacrylate as Skin Adhesive is a good alternative use, showing adequate biocompatibility. Featuring a moderate inflammatory reaction, limited and of short duration dermal level. The scar achieves better cosmetic results regarding the incisions closed with sutures. Consideration should be limited to use low-tension sites not exposed to movements.

KEY WORDS: Rabbit; Healing; Ethyl cyanoacrylate; suture.

\section{REFERENCIAS BIBLIOGRÁFICAS}

Andrade, J. N.; Cuevas, S. E.; Maniscalco, C. L.; Stefanes, S. A. Junqueira, J. \& Santos, P. P. Uso do etil-cianoacrilato na síntese da parede vascular em cães. Ars. Veterinaria, 17(3):172-6, 2001.

Benavides, J. Reparación de heridas cutáneas. Rev. Asoc. Col. Dermatol., 16(1):29-35, 2008.

Borba, C. C.; Roubaud Neto, E.; Val, R. L. R.; Borba Jr, C. O.; Soufen, M. A.; Francisco Neto, A. \& Sakotani, A. Y. Uso do cianoacrilato na síntese da pele de ratos: trabalho de pesquisa experimental. Acta Cir. Bras., 15(1):48-54, 2000.
Chicas, J. Tiempo de cicatrización por segunda intención de piel, en ratas bajo tratamiento con dexametasona; comparando la criocirugía con la cirugía convencional. Tesis presentada para la obtención del título de Médico Cirujano. Facultad de Medicina. Universidad Francisco Marroquin, 2004.

Dalvi, A. A.; Faria, M. M. \& Pinto, A. A. Non-suture closure of wound using cyanoacrylate. J. Postgrad Med., 32:97-100, 1986.

Cardoso, C. A. Análise histomorfométrica do reparo tecidual após laserterapia e aplicação de adesivo. Tese de Mestrado 
VÁSQUEZ, B.; SCHENCKE, C.; RODRÍGUEZ, C.; VEUTHEY, C. \& DEL SOL, M. Comparación entre Etil-cianoacrilato y sutura convencional en el cierre de incisiones de piel de conejo (Oryctolagus cuniculus). Int. J. Morphol., 30(3):797-802, 2012.

em Engenharia Biomédica. Instituto de Pesquisa y Desenvolvimiento. Universidade do Vale do Paraíba, (2003).

Freitas-Junior, R.; Paulinelli, R. R.; Rahal, R. M.; Moreira, M. A.; Olivera, E. L.; Aiko, K. F. \& Approbato, M. S. Estudo experimental comparando o uso do 2-octil cianoacrilato ao nylon 40 na sutura da pele. Rev. Col. Bras. Cir., 35(3):194-8, 2008.

Faion, A. G. Etil-2-Cianoacrilato como selante em coto cecal de Rattus norvegicus albinus submetidos a ressecção parcial. Teses de Mestrado em Ciências da Saúde. Faculdade de Medicina. Universidade Federal do Uberlândia, 2009.

Consenso sobre cicatrización de heridas. Sociedad Argentina de Dermatología, 2008. www.campusveterinario senweb.com

Montanaro, L.; Arciola, C. R. Cenni, E. Ciapetti, G.; Savioli, S.; Filippini, F \& Barsantic, L. A. Citotoxicity, blood compatibility and antimicrobial activity of two cyanoacrylate glues for surgical use. Biomaterials, 22(1):59-66, 2001.

Montes, A.; Cruz, R. \& Chávez C. Efectividad entre el adhesivo tisular (cianoacrilato) vs sutura convencional para el cierre y reparación de heridas superficiales provocadas por trauma. Archivo de Medicina de Urgencia de México, 1(2):51-4, 2009.

Orozco-Razón, L. F.; Millán-Gerrero, R. O. \& Vera-Rodríguez, S. E. Cianoacrilato comparado con cirugía tradicional en el cierre de heridas en zonas libres de tensión. Gac. Méd. Méx. 138(6):505-9, 2002.

Ramírez, G. Fisiología de la cicatrización cutánea. Revista Facultad de Salud, 2(2):69-78, 2010.

Reyes, J. O.; Ramírez, A. T. \& Vejar, I. Empleo de 2-cianoacrilato de n-butilo en heridas libres de tensión. Medicina oral, 12(1):18-23, 2010.

Sangwan, V.; Singh, A. P. \& Kumar, A. Comparative clinical studies on closure of skin incision with tissue adhesive (Iso amyl-2 cyanoacrylate) or sub-cuticular sutures in dogs. Haryana Vet., 42:45-7, 2003.

Saska, S.; Minarelli, A. M. \& Hochuli-Vieira, E. Adesivos à base de cianoacrilato para síntese de tecido mole. An. Bras. Dermatol., 84(6):585-92, 2009.

Shivamurthy, D. M.; Singh, S. \& Reddy, S. Comparison of octyl2-cyanoacrylate and conventional sutures in facial skin closure. Natl. J. Maxillofac. Surg., 1(1):15-9, 2010.

Souza, S. C.; Oliveira, W. L.; Soares, D. F. O. S.; Briglia, C. H.; Athanázio, P. R.; Cerqueira,, M. D.; Guimarães, P. H. \& Carreiro, M. C. Comparative study of suture and cyanoacrylates in skin closure of rats. Acta Cir. Bras., 22(4):309-16, 2007.

Souza, S. C. \& Briglia, C. H. Comparative study of the use of ethyl cyanoacrylate adhesive and intracutaneous suture for cutaneous escisión closure. Rev. Bras. Cir. Plást., 26(4):566-72, 2011.
Toriumi, D. M.; Raslan, W. F.; Friedman, M.; Tardy, M. E. Histotoxicity of cyanoacrylate tissue adhesives: a comparative study. Arch Otolaryngol Head Neck Surg., 116(5):546-50, 1990.

Vasconcellos, C. H.; Matera, J. M. \& Dagli, M. L. Clinical Evaluation of Random Skin Flaps Based on the Subdermal Plexus Secured with Sutures or Sutures and Cyanoacrylate Adhesive for Reconstructive Surgery in Dogs. Veterinary Surgery, 34(1):59-63, 2005.

Dirección para correspondencia:

Bélgica Vásquez P.

Facultad de Ciencias de la Salud

Universidad Autónoma de Chile

Sede Temuco - CHILE

Email: bvasquez@uautonoma.cl

Recibido : 02-03-2012

Aceptado: 24-06-2012 